\title{
Recruiting young people with a visible difference to the YP Face IT feasibility trial: a qualitative exploration of primary care staff experiences
}

\section{Claire Hamlet, Heidi Williamson and Diana Harcourt}

Centre for Appearance Research, University of the West of England, Bristol, UK

\begin{abstract}
Background: Qualitative research methods embedded within feasibility trials are of significant value as they can provide important information for a definitive trial, often unable to be fulfilled by quantitative methods alone. In addition, such information can aid researchers running other trials or evaluating interventions on a similar topic. Aim: This study aimed to explore GP and nurses' experiences of recruiting to a trial exploring the feasibility of evaluating YP Face IT, a novel online psychosocial intervention to support young people with appearance-altering conditions. Methods: During the recruitment period, a focus group with participating GPs and nurses explored recruitment challenges. In addition, at the end of the recruitment period, telephone interviews were conducted with eight GPs and nurses involved in recruiting to the study, in order to inform a definitive trial of YP Face IT. Transcripts were subjected to thematic analysis. Findings: Despite reporting that the study was valuable and interesting, interviewees struggled to recruit in-consultation. They appeared to lack confidence in raising the sensitive issue of a visible difference and adopted strategies to avoid mentioning the topic. Participants felt the nature of the target population, as well as pressures of the primary care environment presented challenges to recruitment, but welcomed YP Face IT as an intervention that could address unmet support needs. Primary care staff may benefit from training to help them raise the subject of a visible difference with young people in order to identify those that require additional support.
\end{abstract}

Key words: adolescent health; qualitative research; trial recruitment; visible difference; YP Face IT

Received 16 January 2017; revised 6 April 2017; accepted 14 July 2017; first published online 14 August 2017

\section{Introduction}

Estimates suggest one in 44 individuals in the United Kingdom have a visible difference/disfigurement (Changing Faces, 2012) resulting from craniofacial (eg, cleft lip/palate) or skin conditions (eg, psoriasis), injuries or medical/surgical treatments (eg, after

Correspondence to: Claire Hamlet, Research Associate, Centre for Appearance Research, University of the West of England, Coldharbour Lane, Bristol, BS16 1QY, UK. Email: claire. hamlet@uwe.ac.uk cancer diagnosis or burn injury). Irrespective of the severity, cause or site of a difference, around $30-50 \%$ of young people affected can experience psychosocial difficulties including social stigma, anxiety and body image dissatisfaction (Rumsey and Harcourt, 2007). As evidence-based psychosocial support for this group is scarce (Jenkinson et al., 2015), an online intervention for 12-17 year olds was developed in collaboration with clinical experts and young people. As an adjunct to medical treatment, YP Face IT (YPF) aims to support young people struggling with the psychosocial consequences of 
appearance-altering conditions (www.ypfaceit. co.uk). Coping strategies, based on social interaction skills training and cognitive behavioural therapy, are delivered via multi-media over seven weekly sessions (see Williamson et al., 2015; 2016 for further details). Its online delivery is particularly amenable to adolescents, who are typically comfortable seeking health-related support and information via the Internet and prefer self-help as treatment (Farrand et al., 2006). Additionally, online support can overcome barriers such as selfconsciousness and poor help-seeking behaviour that prevent young people with a visible difference accessing traditional face-to-face support (Williamson et al., 2015).

General practitioners (GPs) are 'gatekeepers' for healthcare in the United Kingdom. As the first port of call for most patients requiring psychological intervention, they are responsible for assessing and referring them for appropriate support, whilst being mindful of financial imperatives (Forrest, 2003). With greater demand for self-management tools that can potentially complement, replace or prevent the need for costly alternatives (Barak and Grohol, 2011), GP practices are increasingly asked to host research evaluating web-based self-help interventions to address conditions such as obesity (Yardley et al., 2014), depression (Wright et al., 2014) and in this case, appearance-related distress (Williamson et al., 2016). However, research in primary care can be challenging, with projects often hindered by poor recruitment due to competing demands (Lord et al., 2016). Given the current drive to improve recruitment in these settings, employing complementary qualitative research methodologies to inform definitive trials is becoming increasingly important (van Staa et al., 2014). This study therefore aimed to explore, and learn from, GP and nurses' experiences of recruiting to the YPF feasibility trial: the first within primary care to focus on supporting those with a visible difference via an online intervention.

\section{Methods}

\section{Setting}

The YPF feasibility trial recruited 15 GP practices in the south west of England to establish the viability of conducting a randomised-controlled trial to evaluate online support for visible differences as an adjunct to treatment as usual (TAU) in a primary care setting. Due to anticipated challenges of practice staff broaching the subject of appearance (Williamson and Rumsey, 2016), training was provided on the psychological impact of living with a visible difference and how to raise the subject of appearance anxiety with eligible patients. As GPs were asked to supervise their patients randomised to YPF, training was also provided on how to access and review their patients' data (eg, patients reflections on their experiences) on the website (this took $5 \mathrm{~min} /$ completed session) to check for safeguarding concerns (eg, disclosure of self-harm or abuse) and manage these in line with the study's safeguarding policy. Practices sent targeted invitations to 12-17 year olds identified as having an appearancealtering condition on their database and the research team took consent. In response to recruitment difficulties, four additional practices trialled a generic mail-out approach to all 12-17 year olds on their practice list. In total, 47 young people were randomised $(\mathrm{YPF}=23$; $\mathrm{TAU}=24)$. Four were recruited in-consultation, 17 via targeted invitations, 15 via generic mail-out and 11 via other means (eg, charities). Their differences included facial palsy, scarring, and skin and craniofacial conditions.

\section{Data collection}

In response to initially slow recruitment, the research team invited GPs/nurses from each site to attend a focus group partway through the recruitment period. Rather than imposing a focus group schedule, the group were asked to identify and discuss the barriers and facilitators to recruiting young people with a visible difference into the study. Five GPs and one nurse (three males) from six practices participated. In addition, at the end of the recruitment period, GPs/nurses responsible for recruitment (and screening patients identified through database searches) were invited to participate in face-to-face or telephone interviews about their experiences of being involved in the study. Six GPs and two nurses from seven practices (four males; primary care research experience of 3-36 years) participated in semi-structured, audiorecorded telephone interviews. These lasted up to $30 \mathrm{~min}$ and were conducted by the lead author. An interview schedule, developed to assist with assessing the feasibility of conducting a definitive trial of the intervention, focussed on experiences 
of recruiting to the study and supervising patients' use of YPF. Overall, qualitative data was gathered from nine different GPs and two nurses, representing 10 different practices. One GP and one nurse took part in both the focus group and interviews.

\section{Analysis}

The focus group and interviews were transcribed verbatim, anonymised and analysed together by the lead author using inductive Thematic Analysis, whereby coding and theme development was directed by the content of the data rather than existing concepts or ideas (Braun and Clarke, 2006). Transcripts were coded and themes were identified and clustered with a descriptive summary provided for each. To address reliability, the second author reviewed all transcripts; findings were discussed and consensus reached.

\section{Results}

The main themes to emerge from the analysis were as follows:

\section{A 'conspiracy of silence'}

This captures the implicit assumptions participants inadvertently drew upon when deciding on the suitability of patients for the study. Beliefs that young people would prefer not to discuss appearance-related concerns with their GP and that young people perceive a GP's role and responsibility to provide solely medical solutions appeared to perpetuate a 'conspiracy of silence' for raising the topic of visible difference.

\section{A 'sensitive topic'}

Participants acknowledged that raising the subject of a visible difference, whether in writing or in person, was challenging. Without seeking confirmation, some staff assumed that young people would not want to be reminded about their difference through being invited to take part.

'some of them had stuff like a cleft palate, they actually wanted to forget they had a cleft palate; they didn't want to be approached about it as they had been through the official channels originally and were trying to forget about it rather than again counselled about it'.

(GP 2)

Perhaps demonstrating a reluctance to address the topic directly, participants adopted strategies to enable them to invite with greater ease. For example, it was reported as easier to broach if the young person presented because of the condition that affects their appearance rather than something unrelated ' [...] if they came in with something they were embarrassed about, it's easy to chat, it can be a bit more tricky if you are picking something up' (GP 4). Some avoided referring to the visible difference at all. 'I think we are getting more comfortable just making it [the research] more generic I suppose, sort of not to highlight the condition we are suggesting it for' (GP 5)'. Finally, one GP tried to appeal to young people's altruistic side 'I think that helped, offering somebody the chance to do something, to say that you can help other people' (GP 2).

In contrast, difficulties introducing the study were refuted by others, who felt that discussing a visible difference should be no more challenging than other sensitive topics.

'It is not usually a difficult conversation. It shouldn't be any more difficult than having a conversation with an adult about their diabetes'.

(Nurse 2)

'The message is that often people aren't as shocked or upset as you may think, it's better to say something, otherwise there's this continuing conspiracy of silence'

(GP 1, focus group)

\section{'GPs provide medical treatments'}

Participants' perception that young people visit their GP for a medical 'cure' may have functioned as a barrier to raising the topic of psychological support during consultations. They expressed a conflict between meeting patients' expectations to prescribe only medical treatments and raising the topic of YPF, an intervention offered as an adjunct to available medical treatments (which, for many conditions, may have limited effectiveness) that advocates adapting to and coping with a visible difference psychosocially.

"What they were there for was "how do I get rid of my acne?", "how do I treat my eczema?",

Primary Health Care Research \& Development 2017; 18: 541-548 
rather than me saying "well have you considered the psychological implication?",

Participants suggested that young people have no expectations of GPs offering psychological or emotional support, which made it harder to encourage them to participate.

'there was a little bit of convincing [needed that] this would benefit. ...one of the reactions was being wary of this rather than thinking "oh wow, this is something I have been looking for". That may be a reflection of the fact that it may not be on their radar and that they didn't know it was out there'

(GP 3)

\section{Barriers of the primary care environment}

This theme captures narratives relating to the suitability of primary care as a setting for the study, primarily due to time constraints and the nature of the trial's target population which made it difficult to identify participants in-consultation.

\section{'It's hard when you're fighting time'}

Despite practices' enthusiasm and expressed commitment to the study, many of the participants reported barriers to prioritising the study, which made recruitment challenging. Barriers included time-limited consultations, a high workload, competing studies and challenges in engaging colleagues in the recruitment process.

'What we didn't get any response to really was the GPs considering it as an option. I don't know whether it wasn't at the forefront of their minds at this time [...] or whether generally speaking it was hard to consider one or more studies at a time if you are running a surgery. Or whether it was because of the subject matter itself, I think it's hard to ascertain'.

(Nurse 2)

'With the best will in the world, it's hard as you are fighting time and you can't remember stuff.

(GP 1)

The limited time to dedicate to research may have led to reports that although supervising patients' use of YPF was simple, 'yes it was really quick and interesting reading their responses'

Primary Health Care Research \& Development 2017; 18: 541-548
(Nurse 2), there were also concerns about taking responsibility for this: 'because notoriously with these things it is the risk of missing something, that is always a source of concern' (GP 3).

In addition, one GP appreciated the recruitment focus group, which permitted them time to consider the study once it was in progress, share their experiences with others in the same position and become re-enthused.

'When you start a study its worth going back and doing some aftercare because having a meeting and a pow wow about something once you are doing it is better, it makes a huge difference to the people recruiting and enthuses them again'.

(GP 2).

Young people with a visible difference as infrequent attenders

Despite initial optimism in recruiting four young people per practice, participants reported that, on reflection, the nature of the target population was a significant barrier to recruitment. They felt that teenagers, especially those with a visible difference, rarely present in primary care. This not only led to reduced opportunities to identify participants but also resulted in the study slipping from their mind or being surpassed by studies that sought participants with more common conditions.

'We had a thing on sore throats in winter, and every surgery you can get a sore throat and it's easy to remember [...]. But if it's something that is uncommon and you are recruiting for god knows how many things as well, it's really hard to remember'.

(GP 1)

Furthermore, young people who did present were often accompanied by a parent. Some participants felt this dynamic deterred the young person's full engagement in the consultation and their consideration of the study.

'It has made me realise that a lot of the barriers to recruitment are about adults and not younger people [...] his Mum was quite assertive and it was very difficult to talk to him and I think if she hadn't been there he would have gone for it. She decided she did not have time to fit it into her busy schedule'.

(Nurse 2) 
Opinions that teenagers with a visible difference are rare in primary care could have been influenced by expectations that severity (as indicated, eg, by the extent of scarring) predicts distress, potentially obscuring staff judgement on suitable participants. However, as their experience grew, some were challenging this perspective.

'I suppose in hindsight one wonders whether there were clearly some patients with mild eczema or acne [...] who had equally valid psychological issues in so far as their perception'.

(GP 3)

On the whole, participants conveyed an understanding that they should seek each young person's perspective on the impact of their condition and their need for support, rather than making that decision for them.

'Because it's about finding out what matters to the person isn't it? As some people have terrible things wrong about them and are actually quite chilled and don't mind'.

(GP 2)

\section{A welcome study}

Despite difficulties with recruitment, participants identified various motivations for taking part in the study. They felt it was unique in focussing on teenagers, a group less often involved in research.

'I think it's quite nice, perhaps more difficult, but it's nice to do something that involves teenagers, as there is not a great deal'.

(Nurse 1)

Others reported a personal interest in the topic, which increased its pertinence and served as a motivator for recruitment.

'I have a particular interest in it, because my son suffers very badly from psoriasis [...] I think when approaching young people, you need to feel positive that it is a good study [...] then you can communicate the value of taking part really easily'.

(Nurse 2)

Participants expressed frustrations with the lack of support and referral pathways for patients experiencing appearance-related distress and welcomed the intervention as potentially addressing this. In addition, its online delivery was deemed useful as it could provide immediate support:

'CAMHS [Child and Adolescent Mental Health Services] is awful as they are only really interested in people who have severe problems and they have to wait ages to be seen and with teenagers you really want to act then and do something instantly. That's why doing something online is good [...] they are always on their device'

(GP 2)

On the whole, participants reported the study had raised their awareness of the psychosocial impact of a visible difference, increasing the likelihood that they would consider or explore emotional support in addition to medical treatment in the future.

'So before the study I have to say that if someone came to see me with acne, I would have focused absolutely primarily on the ranges of treatment [...] but since the study I am certainly much more inclined to ask about whether there are any issues at school or psychological issues'.

(GP 3)

\section{Discussion}

Despite modest targets and utilising additional recruitment strategies, only two practices in this feasibility trial met their target. In-consultation recruitment was particularly ineffective. Participants reported that teenagers infrequently consulted in primary care, which made it hard to recruit to the study, a finding supported by other research with this age group (Iliffe et al., 2008). Despite training and guidance on how to sensitively discuss the study with patients, participants seemed hesitant approaching the topic directly concerns previously reported when dealing with other sensitive topics such as self-harm (Fox et al., 2015). They adopted strategies such as only broaching the subject if appearance-related concerns were the reason for the consultation, which is problematic as young people rarely initiate a request for psychological support (YMCA, 2016), 
often waiting for the GP to raise the topic (Mauerhofer et al., 2009). If health professionals struggle to normalise and validate appearancerelated concerns (an aim of current societal interventions, eg, Stice et al., 2000), this 'conspiracy of silence' may reinforce young people's beliefs that appearance concerns are inconsequential (Williamson et al., 2010). This serves to further stigmatise the topic and prevent young people seeking support. The importance of health professionals broaching the subject is illustrated by a member of the study's public and patient involvement group: 'I ask that GPs are as brave as their patients and find a way to offer the benefits of YP Face IT. I recognise appearance can be a delicate subject for GPs, as well as their patients, but had YP Face IT been available and offered as an early intervention, I feel my road back to confidence would have been a lot quicker and easier'.

A number of the study's findings are applicable to diverse areas of primary care research. First, staff with a genuine or personal investment in the topic area are likely to act as champions and put more effort into promoting the study (Bell-Syer and Moffett, 2000). In addition, interventions with the potential to benefit the practice or patient population are favoured (Moore and Smith, 2007), as with this study where YPF was seen as a potential means of offering support to a group currently underserved. This study verified reports that conducting primary care research remains challenging, potentially identifying the need for a cultural shift that permits time and space to engage in research, particularly as identifying new, cost-effective interventions are important in the National Health Service (NHS) (Mason et al., 2007). With indications that parents can act as gatekeepers, and since research indicates that adolescents find it easier to broach sensitive topics if they consult alone (Bravender et al., 2004), future recruiters may consider the benefits of providing young people with additional opportunities to voice their interest in research/interventions when joined by their parents. Finally, patient uptake into research evaluating psychosocial interventions might be enhanced if GPs communicate whether or not they will replace the medical treatment that patients expect.

In addition to informing the design of a definitive trial to determine the effectiveness of YPF, this study highlights the potential need for training to educate primary care staff to broach the topic of a visible difference confidently, both within and outside the parameters of research. Training, with a particular focus on how to talk to young people who might be experiencing appearance concerns, could facilitate doctor-patient communication about the psychosocial challenges of living with a condition or injury that alters appearance and, in turn, patient disclosure.

\section{Limitations}

This qualitative study involved a small sample of GPs and nurses who may have self-selected to take part because they were interested in the topic, more motivated or had time to share their experiences. Therefore, we cannot claim that their experiences are representative of primary care staff more broadly, yet still the study raises important issues for consideration in future research. In addition, only two nurses contributed to the data, which did not allow us to explore any differing perspectives between nurses and GPs. This is a worthwhile area for future enquiry, particularly when exploring recruitment to trials concerning a sensitive topic. The interviewer was also a researcher known to the participants, which might have affected their openness, despite being assured of confidentiality and anonymity.

\section{Conclusion}

Recruiting to the YP Face IT feasibility trial was challenging and required flexibility and creativeness to facilitate open discussions with recruiting practices. This research highlighted noteworthy recruitment barriers, including difficulties raising the subject of appearance, the pressures of primary care and young people's perceptions regarding the role of a GP. Despite this, positive ways of navigating these challenges were reported such as normalising and validating the topic of appearance, a particularly important undertaking to help identify those that require additional support.

\section{Acknowledgements}

The authors thank all the GPs and nurses who took the time to participate in the focus group and 
interviews, the participating GP practices and the West of England Clinical Research Network for supporting recruitment. The authors also thank the study management group and steering committee for their involvement and Zoe Guest and Laura Bishop for their assistance with interview transcription.

\section{Authors' Contribution}

C.H. wrote the first draft. C.H. and H.W. analysed the data. All authors were involved in interpreting the data and preparation of the manuscript.

\section{Financial Support}

National Institute for Health Research grant number, PB-PG-1112-29014. This Report is independent research funded by the National institute for Health Research's Research for Patient Benefit. The views expressed in this publication are those of the author(s) and not necessarily those of the NHS, the National Institute for Health Research or the Department of Health.

\section{Ethical Standards}

This research received a favourable ethical opinion from the NRES Committee South WestFrenchay (reference 14/SW/0058).

\section{Conflicts of Interest}

None.

\section{References}

Barak, A. and Grohol, J.M. 2011: Current and future trends in internet-supported mental health interventions. Journal of Technology in Human Services 29, 155-96.

Bell-Syer, S.E. and Moffett, J.A.K. 2000: Recruiting patients to randomized trials in primary care: principles and case study. Family practice 17, 187-91.

Braun, V. and Clarke, V. 2006: Using thematic analysis in psychology. Qualitative Research in Psychology 3, 77-101.

Bravender, T., Price, C.N. and English, A. 2004: Primary care providers' willingness to see unaccompanied adolescents. Journal of Adolescent Health 34, 30-36.

Changing Faces 2012: The face equality campaign: the evidence. The incidence and prevalence of disfigurement. Retrieved 28 November 2016 from http://admin.changingfaces. org.uk/downloads/FE\%20Campaign, \%20Epidemiology\%202\% 20pages.pdf.
Farrand, P., Perry, J., Lee, C. and Parker, M. 2006: Adolescents' preference towards self-help: implications for service development. Primary Care Community Psychiatry $11,73-79$.

Forrest, C.B. 2003: Primary care gatekeeping and referrals: effective filter or failed experiment? British Medical Journal 326, 692-95.

Fox, F., Stallard, P. and Cooney, G. 2015: GPs role identifying young people who self-harm: a mixed methods study. Family Practice 32, 415-19.

Iliffe, S., Williams, G., Fernandez, V., Vila, M., Kramer, T., Gledhill, J. and Miller, L. 2008: General practitioners' understanding of depression in young people: qualitative study. Primary Health Care Research \& Development 9, 269-79.

Jenkinson, E., Williamson, H., Byron-Daniel, J. and Moss, T.P. 2015: Systematic review: psychosocial interventions for children and young people with visible differences resulting from appearance altering conditions, injury, or treatment effects. Journal of Pediatric Psychology 40, 1017-33.

Lord, P.A., Willis, T.A., Carder, P., West, R.M. and Foy, R. 2016: Optimizing primary care research participation: a comparison of three recruitment methods in data-sharing studies. Family Practice 33, 200-4.

Mason, V.L., Shaw, A., Wiles, N.J., Mulligan, J., Peters, T.J., Sharp, D. and Lewis, G. 2007: GPs' experiences of primary care mental health research: a qualitative study of the barriers to recruitment. Family Practice 24, 518-25.

Mauerhofer, A., Berchtold, A., Michaud, P.A. and Suris, J.C. 2009: GPs' role in the detection of psychological problems of young people: a population-based study. British Journal General Practice 59, 308-14.

Moore, M. and Smith, H. 2007: Agreeing to collaborate: a qualitative study of how general practices decide whether to respond positively to an invitation to participate in a research study. Primary Health Care Research and Development 8, 141-46.

Rumsey, N. and Harcourt, D. 2007: Visible difference amongst children and adolescents: issues and interventions. Developmental Neurorehabilitation 10, 113-23.

Stice, E., Mazotti, L., Weibel, D. and Agras, W.S. 2000: Dissonance prevention program decreases thin-ideal internalization, body dissatisfaction, dieting, negative affect, and bulimic symptoms: a preliminary experiment. International Journal of Eating Disorders 27, 206-17.

Williamson, H., Griffiths, C. and Harcourt, D. 2015: Developing young person's Face IT: online psychosocial support for adolescents struggling with conditions or injuries affecting their appearance. Health Psychology Open 2. https://doi.org/ 10.1177/2055102915619092.

Williamson, H., Hamlet, C., White, P., Marques, E.M., Cadogan, J., Perera, R., Rumsey, N., Hayward, L. and Harcourt, D. 2016: Study protocol of the YP Face IT feasibility study: comparing an online psychosocial intervention versus treatment as usual for adolescents distressed by appearance-altering conditions/injuries. BMJ Open 6 , e012423. http://dx.doi.org/10.1136/bmjopen-2016-012423. 
Williamson, H., Harcourt, D., Halliwell, E., Frith, H. and Wallace, M. 2010: Adolescents' and parents' experiences of managing the psychosocial impact of appearance change during cancer treatment. Journal of Pediatric Oncology Nursing 27, 168-75.

Williamson, H. and Rumsey, N. 2016: The perspectives of health professionals on the psychosocial impact of an altered appearance among adolescents treated for cancer and how to improve appearance-related care. Journal of Psychosocial Oncology. https://doi.org/10.1080/07347332. 2016.1247407.

Wright, B., Tindall, L., Littlewood, E., Adamson, J., Allgar, V., Bennett, S., Gilbody, S., Verduyn, C., Alderson-Day, B., Dyson, L. and Trépel, D. 2014: Computerised cognitive behaviour therapy for depression in adolescents: study protocol for a feasibility randomised controlled trial. BMJ Open 4, e006488. https://doi.org/10.1136/bmjopen2014-006488. van Staa, T.P., Dyson, L., McCann, G., Padmanabhan, S., Belatri, R., Goldacre, B., Cassell, J., Pirmohamed, M., Torgerson, D., Ronaldson, S. and Adamson, J. 2014: The opportunities and challenges of pragmatic point-of-care randomised trials using routinely collected electronic records: evaluations of two exemplar trials. Health Technology Assessment 18, 1-146.

Yardley, L., Ware, L.J., Smith, E.R., Williams, S., Bradbury, K.J., Arden-Close, E.J., Mullee, M.A., Moore, M.V., Peacock, J.L., Lean, M.E. and Margetts, B.M. 2014: Randomised controlled feasibility trial of a web-based weight management intervention with nurse support for obese patients in primary care. International Journal of Behavioral Nutrition and Physical Activity 11, 1.

YMCA 2016: A report investigating the stigma faced by young people experiencing mental health difficulties. Retrieved 30 November 2016 from http://www.ymca.org.uk/wp-content/ uploads/2016/10/IAMWHOLE-v1.1.pdf. 